Research article

urn:1sid:zoobank.org:pub:855E32F7-E680-4BC6-A8F4-630ED7FAD5C3

\title{
On the Afrotropical genus Holmelgonia (Araneae, Linyphiidae), with the description of three new species from the Albertine Rift
}

\author{
Benoît NZIGIDAHERA ${ }^{1,3}$ \& Rudy JOCQUÉ ${ }^{2,4}$ \\ ${ }^{1}$ Institut National pour l'Environnement et la Conservation de la Nature (INECN), B.P. 2757 \\ Bujumbura, Burundi. E-mail: nzigidaherabenoit@yahoo.fr \\ ${ }^{2}$ Corresponding author: Royal Museum for Central Africa (MRAC), B-3080 Tervuren, Belgium. \\ E-mail: rudy.jocque@africamuseum.be \\ ${ }^{3}$ urn:Isid:zoobank.org:author:3112C6AF-BB9B-4CF7-B0A6-A709D2473A25 \\ ${ }^{4}$ urn:1sid:zoobank.org:author:CF15016C-8CD1-4C9D-9021-44CA7DC7A5D5
}

\begin{abstract}
Three new species of Holmelgonia were found in the mountain forest of Kibira National Park in Burundi: H. afromontana sp. nov. ( $\overbrace{}^{\top}+$ ), H. bosnasutus sp. nov. ( $\jmath^{\top}+$ ) and $H$. disconveniens sp. nov. $(\hat{O})$. A key to the males in the genus, now containing 17 species, is provided.
\end{abstract}

Key words. Afromontane forest, Burundi, Kibira National Park, identification key, species swarm.

Nzigidahera B. \& Jocqué R. 2014. On the Afrotropical genus Holmelgonia (Araneae, Linyphiidae), with the description of three new species from the Altertine Rift. European Journal of Taxonomy 77: 1-18. http://dx.doi. org/10.5852/ejt.2014.77

\section{Introduction}

Linyphidae have been popular among students of Afrotropical spiders and figure among the better known families of the continent, as far as the species living in the leaf litter layer is concerned. The interest in the family doubtlessly originated in the expertise several taxonomists had acquired in Europe, where the family Linyphiidae is by far the most speciose. Mainly Bosmans, Denis, Holm, Jocqué, Miller, Russell-Smith and Scharff all contributed to a great extent to the knowledge of the family thanks to the experience they had obtained by studying the European linyphiids. By the end of the last century, their extensive studies had yielded enough information about the family to result in an interactive key to the genera (Seyfulina \& Jocqué 2007) and to allow generalisations about the distribution of the family, such as for instance the abundance of montane species (Scharff 1990, 1992, 1993) as compared to the paucity of the family in African lowland habitats and the small size of Afrotropical litter inhabiting linyphiids (Jocqué et al. 2013).

More recently, the fauna of the canopy yielded a high number of unknown linyphiid taxa (see Seyfulina \& Jocqué 2009). Although the interest in Afrotropical linyphiids has apparently dwindled, the recent study by Frick \& Scharff (2013) proves that the interest in Afrotropical linyphiids still slumbers.

The present study aims to stimulate renewed interest in African linyphiids and is the first of a series of papers on linyphiids from the southern part of the Albertine Rift, a mountain range with a particularly 
diverse fauna (Nzigidahera \& Jocqué 2009; Jocqué et al. 2013). It provides an overview of the endemic Afrotropical genus Holmelgonia Jocqué \& Scharff, 2007. The genus, originally described by Holm (1962) as Elgonella, received the replacement name Elgonia Platnick (1986), but eventually became Holmelgonia since Elgonia also turned out to be a homonym (Jocqué \& Scharff 2007).

The genus Holmelgonia contains 14 species (Platnick 2013) and has a wide distribution (see Fig. 1) ranging from Côte d'Ivoire in the west to Kenya and Mozambique in the east. Although most species live at high altitude and have small endemic ranges (Jocqué \& Scharff 1986; Scharff 1993), some of them (e.g., H. holmi Miller, 1970; H. brachystegiae Jocqué, 1981) have a wide distribution at midaltitude.

Here we describe three new species from Burundi and provide a key to all the species for which males have been described.

The collections from which the type material of the new species originates are the result of an extensive sampling campaign carried out by the first author in protected areas of Burundi during three periods in 2003, 2005 and 2008. They covered altitudinal gradients between $1970 \mathrm{~m}$ and $2650 \mathrm{~m}$ above sea level (asl). The discovery emphasizes the biodiversity of the Albertine Rift spider fauna with high numbers of species in many spider groups (Nzigidahera \& Jocqué 2009).

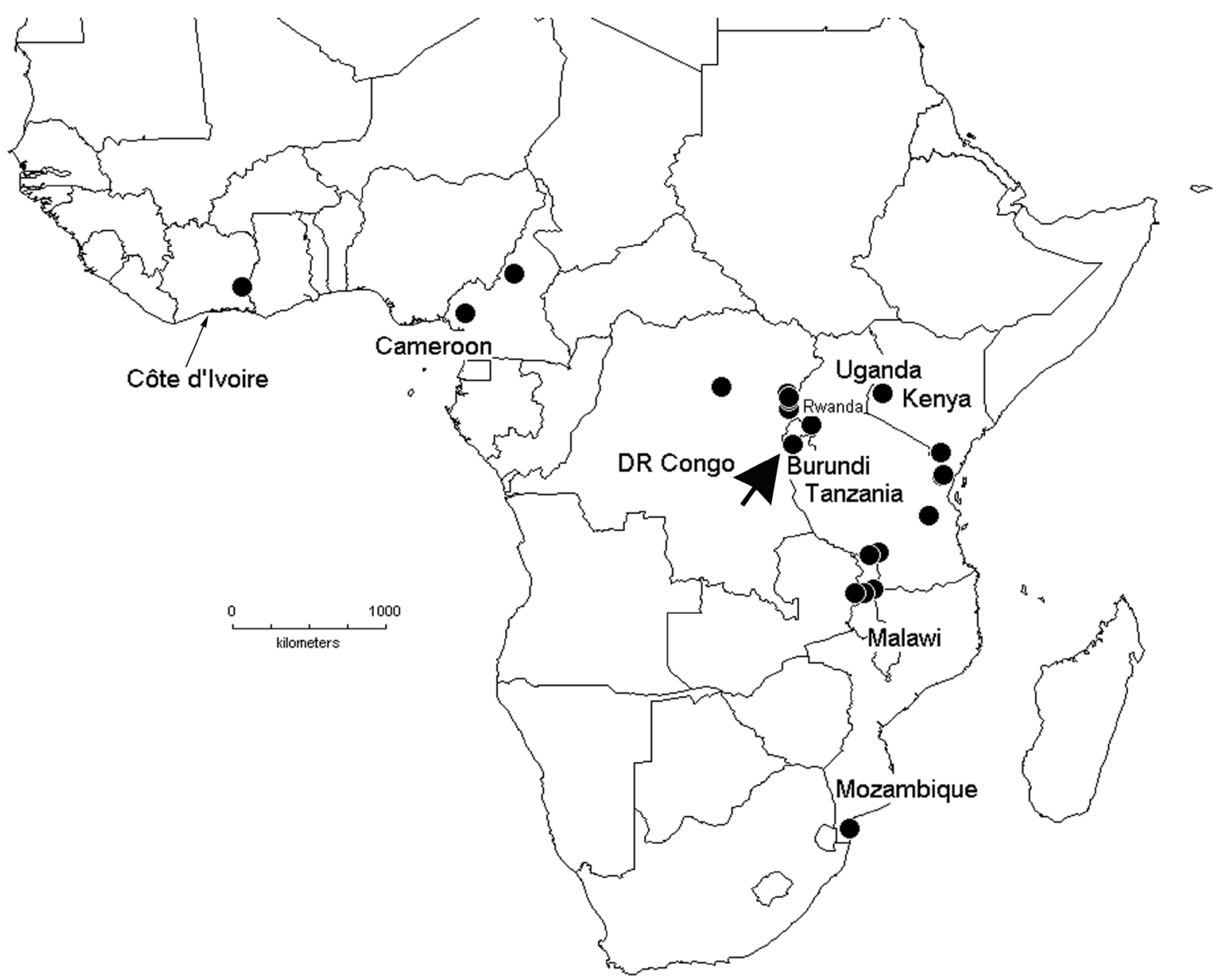

Fig. 1. Presently known distribution of Holmelgonia Jocqué \& Scharff, 2007. Large arrow indicates Kibira National Park, type locality of the species described in this paper. 


\section{Material and methods}

The specimens were collected by the first author in the afromontane forest of Kibira. Primary types are deposited in the Musée Royal de l'Afrique Centrale, Tervuren, Belgium (MRAC), whereas paratypes are divided among MRAC and the Institut National pour l'Environnement et la Conservation de la Nature, Bujumbura, Burundi (INECN). Specimens were observed, drawn and measured with a WILD M 10 stereo microscope. Details of the epigyne and male palps were observed with a Zeiss Stemi 2000 strereo microscope.

Epigynes were detached from the abdomen, cleared with methyl salicylate and temporarily mounted in a mixture of methyl salicylate and cedukol for examination. The same method was used to observe details of the male palp. These structures were photographed with a Leica MZ16 stereo microscope and subject to automontage with the Syncroscopy software. All measurements are in $\mathrm{mm}$. As with the illustrations, they were taken from the holotype and paratypes unless stated otherwise. Coordinates are in decimal format.

\section{Abbreviations}

$\begin{array}{ll}\mathrm{ALE} & =\text { anterior lateral eyes } \\ \mathrm{AME} & =\text { anterior median eyes } \\ \mathrm{AW} & =\text { anterior width } \\ \mathrm{d} & =\text { dorsal } \\ \mathrm{dl} & =\text { dorsolateral } \\ \mathrm{DT} & =\text { diameter of tibia } \\ \mathrm{ED} & =\text { embolic division } \\ \mathrm{Fe} & =\text { femur } \\ \mathrm{L} & =\text { length } \\ \mathrm{MA} & =\text { median apophysis }\end{array}$
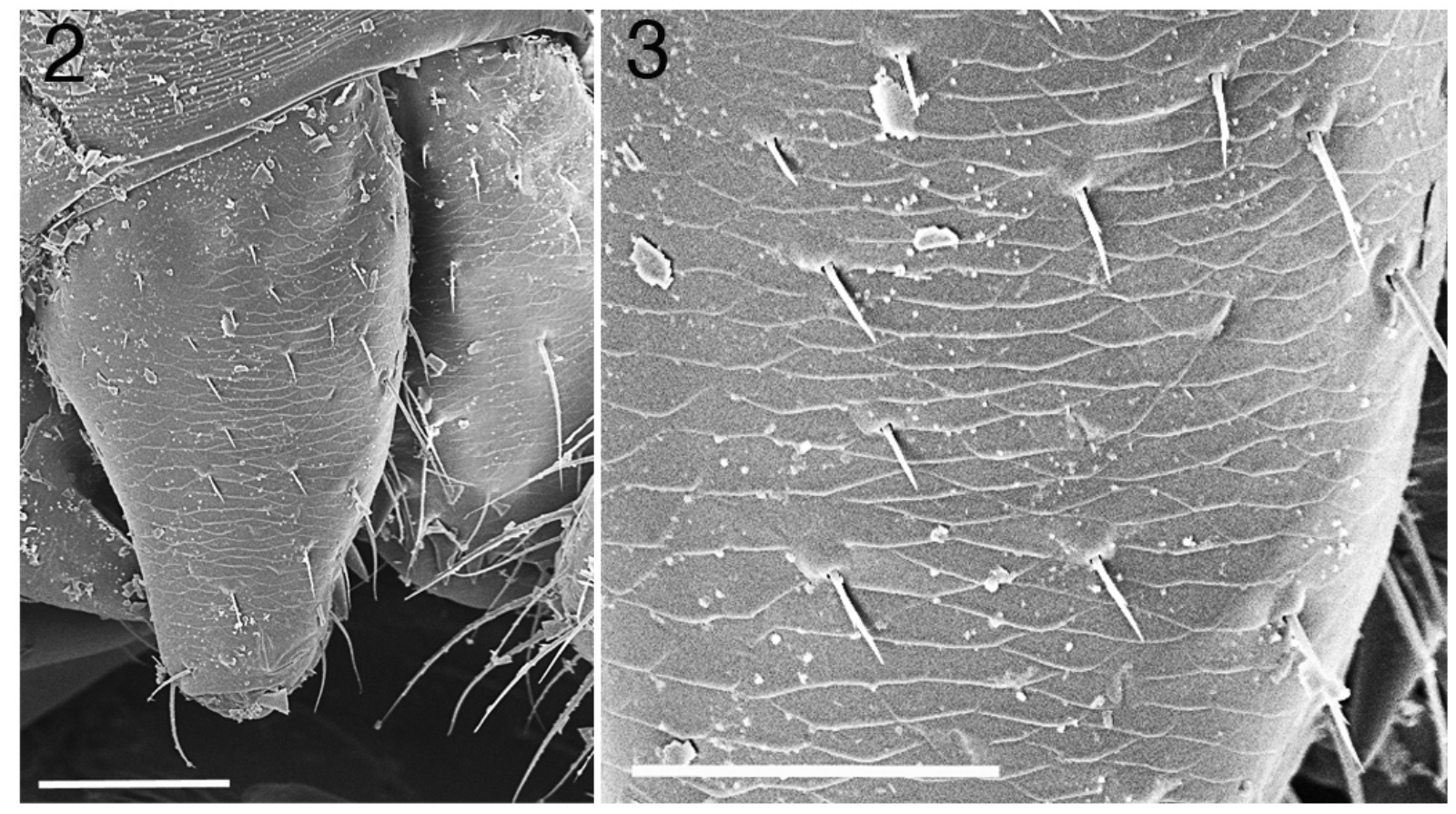

Figs 2-3. Holmelgonia disconveniens sp. nov. ( $\precsim$, MRAC 227149). 2. SEM micrographs of chelicerae, lateral view, showing absence of stridulating ridges. 3. Detail of previous. Scale bars $=100 \mu \mathrm{m}$. 


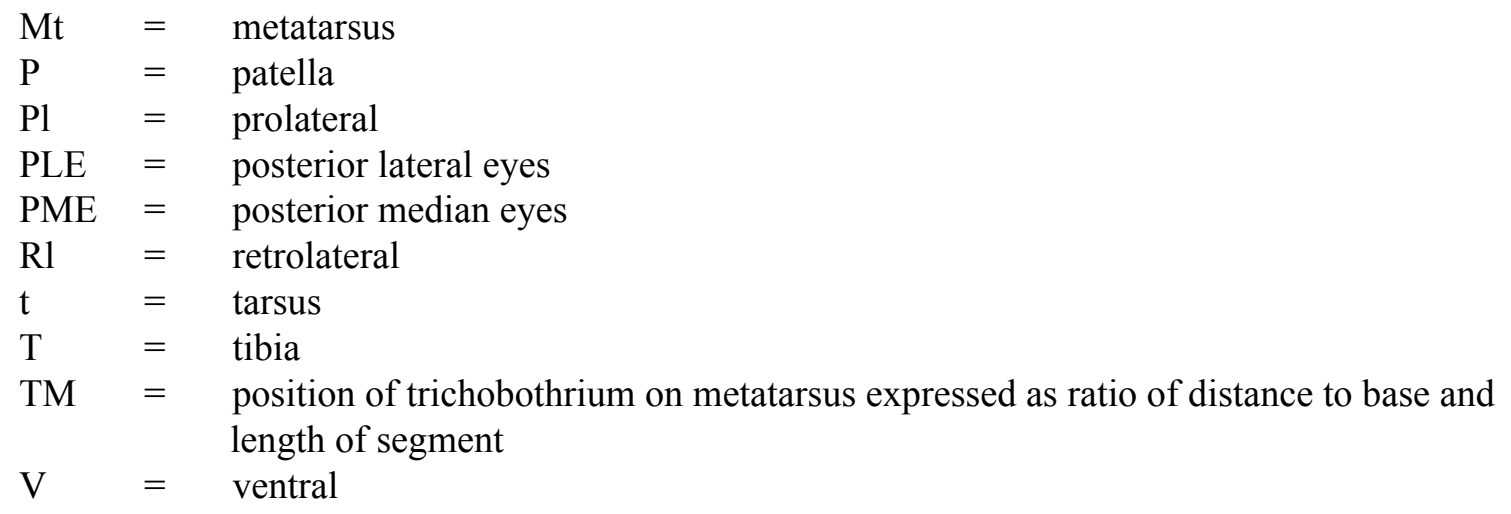

\section{Taxonomy}

Class Arachnida Cuvier, 1812

Order Araneae Clerck, 1757

Family Linyphiidae Blackwall, 1859

Subfamily Erigoninae Emerton, 1882

Holmelgonia Jocqué \& Scharff, 2007

\section{Type species}

Elgonella nemoralis Holm, 1962

\section{Diagnosis}

The generic definition is wide, as the original diagnosis by Holm (1962) had to be enlarged by Jocqué \& Scharff (1986) to accommodate the species from Tanzanian mountain areas. The genus is characterized by the absence of cheliceral stridulating ridges (Figs 2-3); the presence of a double ventral row of setae on the femora; the long tibial spines, two to three times as long as the diameter of the segment; tibia I-IV with 2, 2, 1 and 1 spine, respectively; TMI between 0.32 and 0.7 ; males lack a cephalic lobe; the palpal tibia dorsally produced into a dorsal process (Figs 4-5, 7).

\section{Key to the species of Holmelgonia (males only)}

1. Dorsal tibial apophysis apically without teeth, smoothly rounded and with many long hairs (A) H. afromontana sp. nov. (Burundi)

- Dorsal tibial apophysis apically with teeth, sometimes with many hairs .2
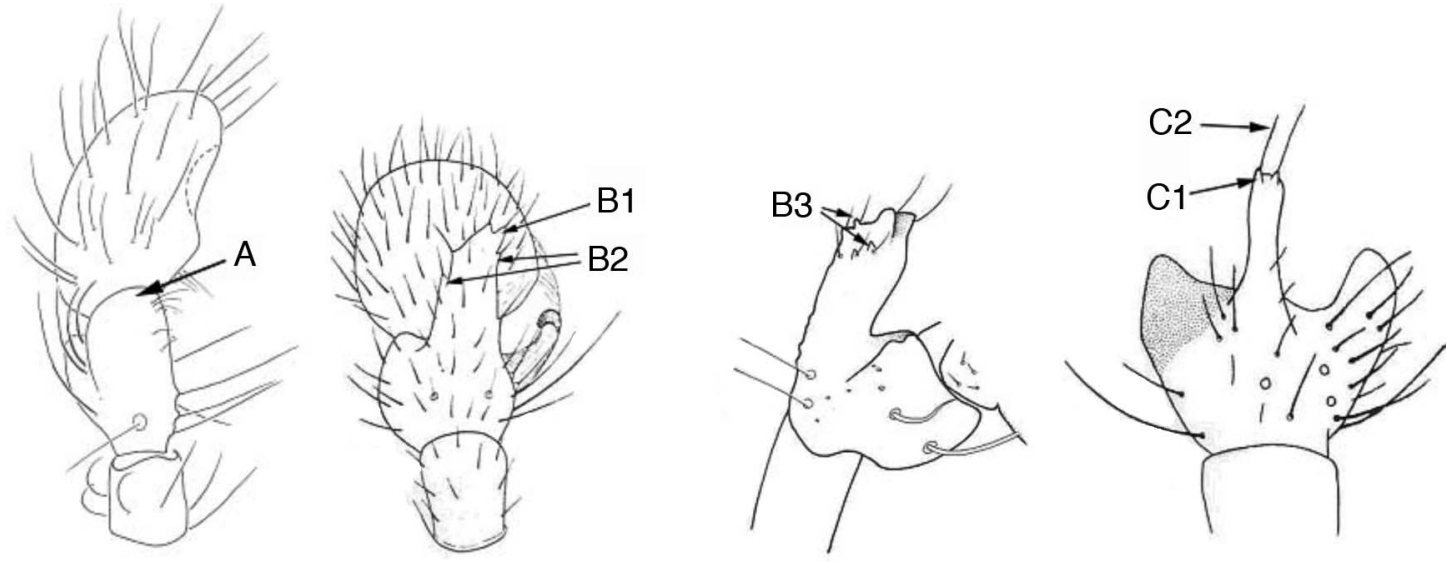
2. Dorsal tibial apophysis apically with two teeth $(\mathrm{C} 1)$, each provided with hair $(\mathrm{C} 2)$ but without teeth or undulation on the margins

- Dorsal tibial apophysis with teeth or undulation on the retrolateral margin (B2, dorsal view) or on distal part (B3, retrolateral view), apart from apical teeth (B1, dorsal view)

3. Dorsal tibial apophysis originating at the proximal base of tibia, straight, pointing upward (D1); retrolateral tibial apophysis absent (D2)

..H. brachystegiae (Jocqué, 1981) (Burundi, Malawi, Tanzania)

- Dorsal tibial apophysis obliquely oriented (E1); retrolateral tibial apophysis conical (E2), toothed

(F) or rounded at the tip $(\mathrm{G})$
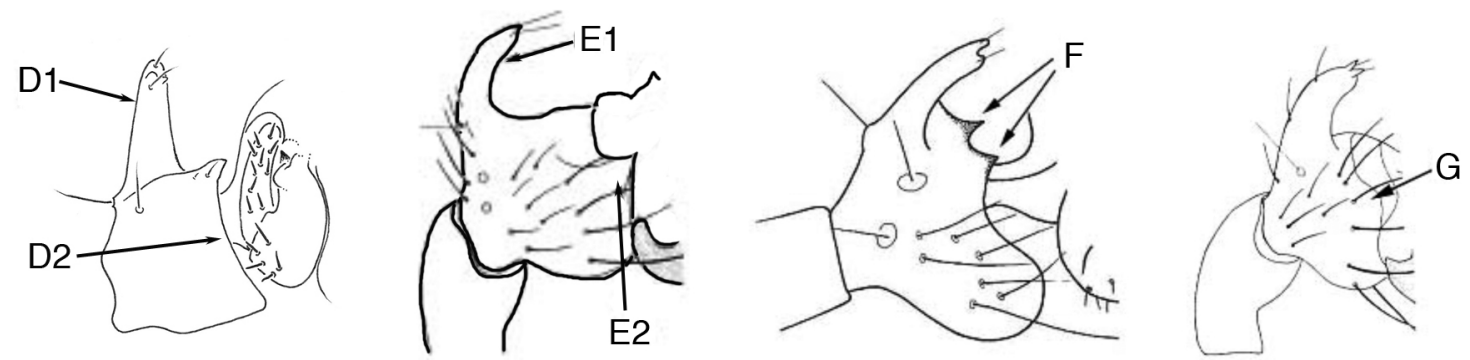

4. Retrolateral tibial apophysis conical (E2) or rounded at the tip (G)

- Retrolateral tibial apophysis with teeth $(\mathrm{F})$

5. Distal arm of paracymbium with indented posterior process $(\mathrm{H})$; embolus semicircular or sickleshaped (I) H. falciformis (Scharff, 1990) (Uzungwa Mts, Tanzania)

- Distal arm of paracymbium with simple posterior process $(\mathrm{J})$; embolus with sharp bend $(\mathrm{K})$... ..H. annemetteae (Scharff, 1990) (Uzungwa Mts, Tanzania)
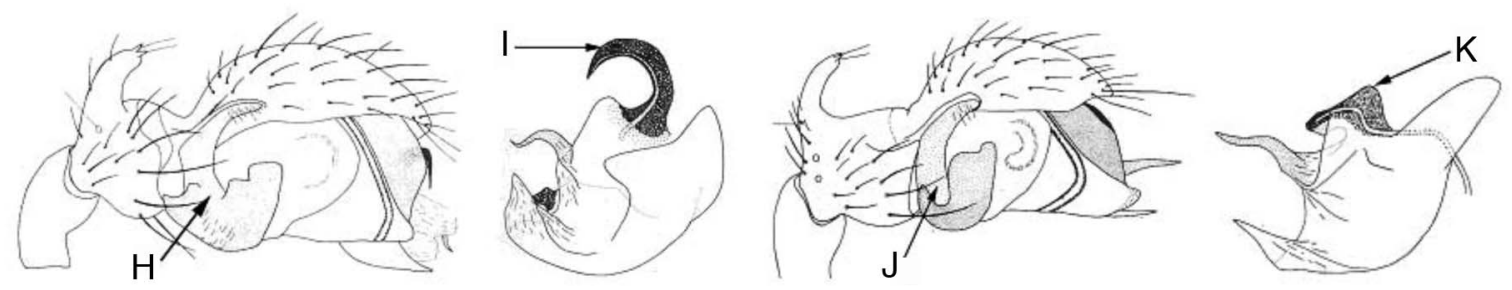

6. Retrolateral tibial apophysis with blackened teeth $(\mathrm{F})$ on the transerverse ridge under the dorsal tibial apophysis H. annulata (Jocqué \& Scharff, 1986) (Uluguru Mts, Tanzania)

- Retrolateral tibial apophysis with a single tooth on the lateral ridge (L) .H. basalis (Jocqué \& Scharff, 1986) (Usambara Mts, Tanzania)

7. Dorsal tibial apophysis with one tooth at the tip (M2); retrolateral tibial apophysis pointed at the tip (M1) ..H. disconveniens sp. nov. (Burundi)

- Dorsal tibial apophysis with two teeth at the tip (B1, B2, C1)

8. Dorsal tibial apophysis with a row of five teeth on the margin $(\mathrm{N})$

H. producta (Bosmans, 1988) (Cameroon)

- Dorsal tibial apophysis with undulation on the prolateral margin (B2) or many small teeth near apical part (B3) 
9. Dorsal tibial apophysis with undulation on the prolateral margin (B2) .10

- Dorsal tibial apophysis with many small teeth on the apical part (B3)
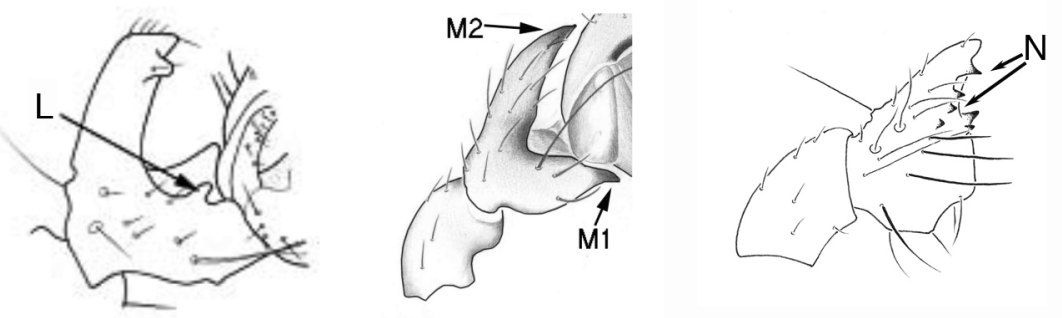

10. Dorsal tibial apophysis with a large prolateral lobe $(\mathrm{O})$

H. holmi (Miller, 1970) (Congo, Cameroon)

- Dorsal tibial apophysis with small prolateral lobes or teeth on the margin (B2)

11. Distal arm of paracymbium rounded at tip (P1), proximal arm broad at base (P2)

..H. bosnasutus sp. nov. (Burundi)

- Distal arm of paracymbium hooked at the tip (Q1), proximal arm narrow at base (Q2) H. nemoralis (Holm,1962) (Kenya)
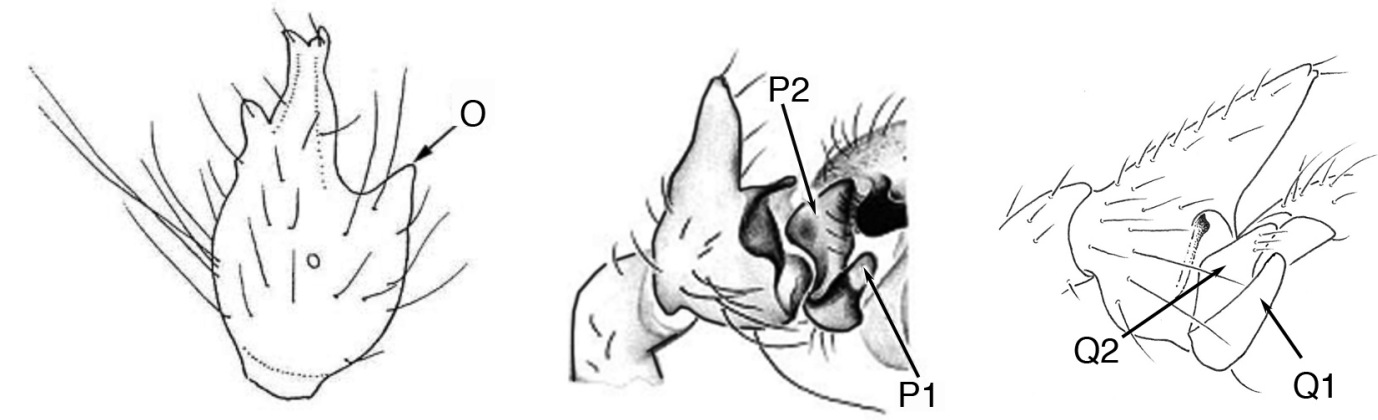

12. Embolic division ventrally with numerous small teeth (R1); frontal embolar apophysis very long (R2), central embolar apophysis very short (R3) and adjacent to embolus (R4)

H. perturbatrix (Jocqué \& Scharff, 1986) (Usambara Mts, Tanzania)

- Embolic division ventrally without teeth; frontal embolar apophysis very long and twisted (S1), central embolar apophysis on lateral side of division (S2) delimiting a concavity provided with numerous small warts (S3) H. projecta (Jocqué \& Scharff, 1986) (Uluguru Mts, Tanzania)
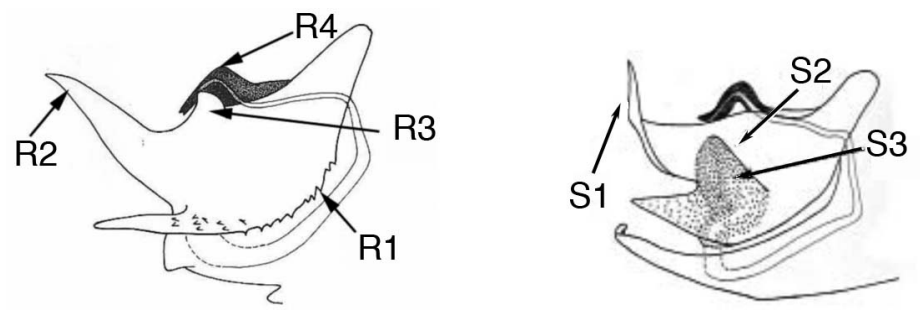

Holmelgonia afromontana sp. nov.

urn:lsid:zoobank.org:act:F482911E-4987-4F7E-BC74-D4101DE9EE29

Figs 4-5, 8, 11-16, 26-29, 39

\section{Diagnosis}

The male is characterized by the dorsal tibial apophysis, which is smoothly rounded at the extremity and has a ventral concavity provided with a short, sharp, tooth-shaped excrescence inside. Proximal 
and distal arms of the paracymbium with short setae, distal arm strongly sclerotized and curved at the tip. The female is recognized by details of the epigyne of which the central depression and the kidneyshaped spermathecae are the most characteristic.

\section{Etymology}

The specific name 'afromontana' refers to the high altitude habitat in which the species was collected.
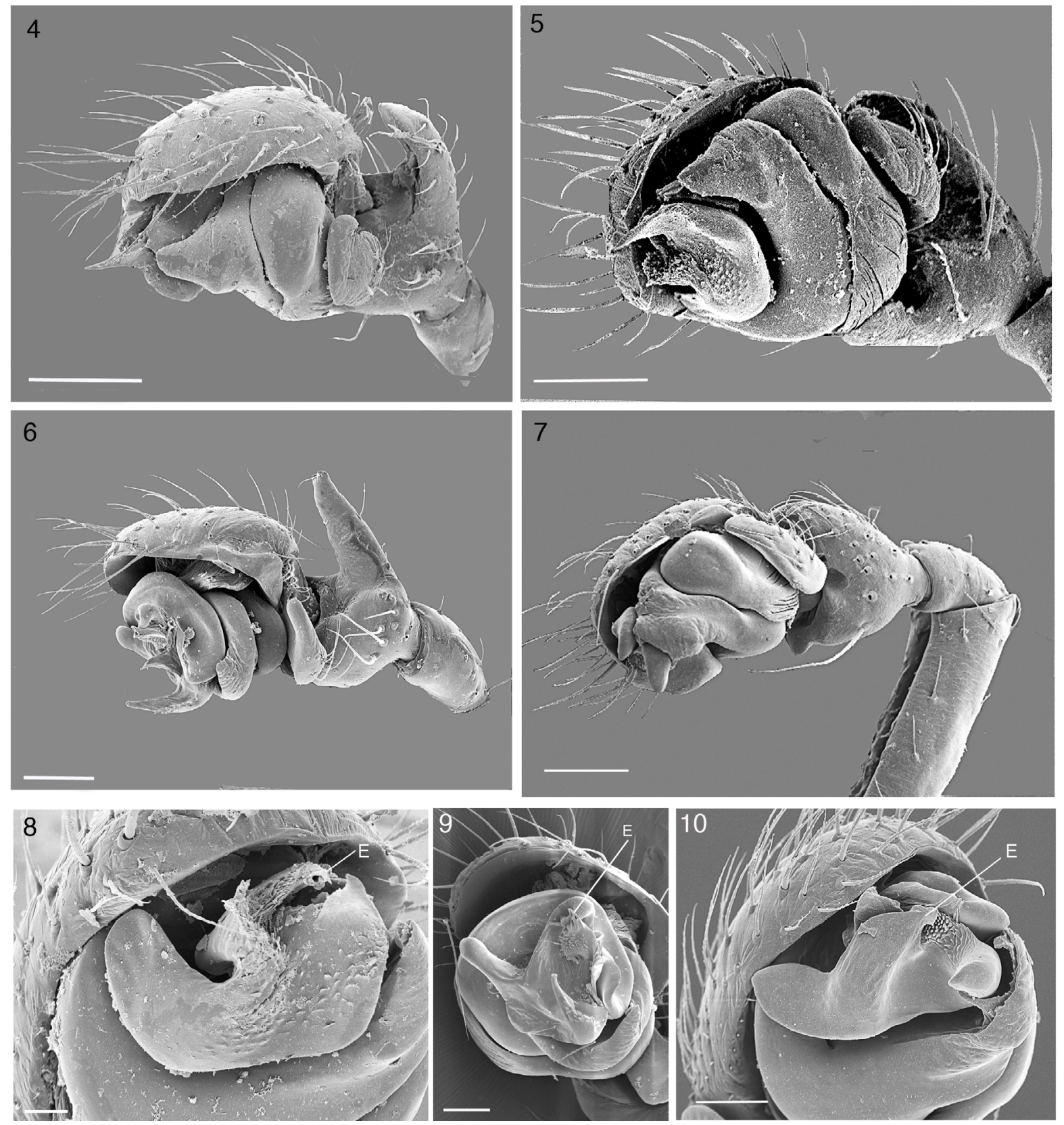

Figs 4-10. SEM micrographs. - 4-5, 8. Holmelgonia afromontana sp. nov. (đ), MRAC 227153). 4. Palp, retrolateral view. 5. As previous, ventral view. 8. Embolic division, frontal view. 6, 9. Holmelgonia bosnasutus sp. nov. ( $\hat{0}$, MRAC 227145). 6. Palp, retrolateral view. 9. Embolic division, ventral view. - 7, 10. Holmelgonia disconveniens sp. nov. ( ${ }^{\lambda}, \mathrm{MRAC} 241448$ ). 7. Palp, retrolateral view. 10. Embolic division, frontal view. Scale bars 4-7 $=100 \mu \mathrm{m} ; 9-10=50 \mu \mathrm{m}$; $8=20 \mu \mathrm{m}$. E: embolus. 


\section{Material examined}

Holotype

BURUNDI: ${ }^{\lambda}$, Parc National de la Kibira, Rwegura, Mt. Musumba, $2.86445^{\circ} \mathrm{S}, 29.49640^{\circ} \mathrm{E}$, site 5, forest with Carapa grandiflora and Polyscias fulva, 25 Jun. 2008, 2252 m, pitfalls, B. Nzigidahera coll. (MRAC 227154).

\section{Paratypes}

BURUNDI: All from Parc National de la Kibira, Rwegura, Mt Musumba, pitfalls, B. Nzigidahera coll. -1 , together with holotype (MRAC 227154). $-2.86435^{\circ} \mathrm{S}, 29.49546^{\circ} \mathrm{E}$, site 6 , forest with Carapa

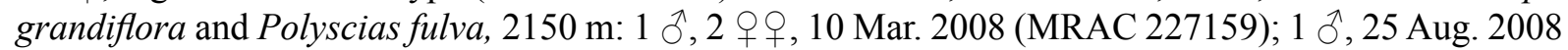

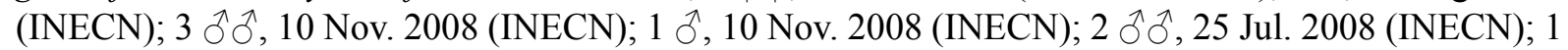
ô, 1 juv., 10 Jul. 2008 (MRAC 227157); 1 q, 25 Mar. 2008 (MRAC 227161). - 2.86445 ${ }^{\circ}$ S, 29.49640 ${ }^{\circ}$ E, site 5, forest with Carapa grandiflora and Polyscias fulva, $2252 \mathrm{~m}: 1$ ก, 10 Apr. 2008 (MRAC

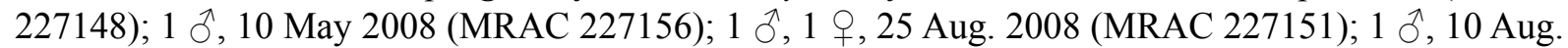

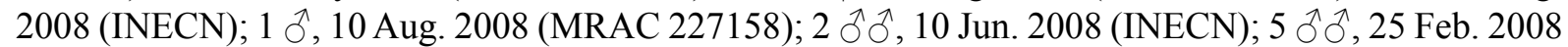

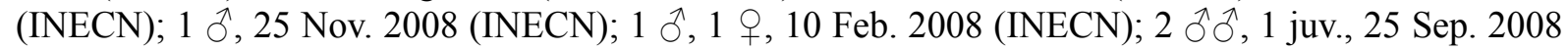
(INECN). $-2.86455^{\circ} \mathrm{S}, 29.50427^{\circ} \mathrm{E}$, site 4, forest with Macaranga neomildbraediana and Polyscias fulva, $2352 \mathrm{~m}: 1$ ㅇ, 25 Jun. 2008 (MRAC 227152); 3 $\widehat{\jmath}, 10$ Feb. 2008 (MRAC 227153); $1 \jmath^{\lambda}, 25$ Feb.

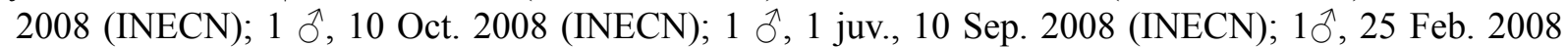
(INECN). $-2.87149^{\circ} \mathrm{S}, 29.49641^{\circ} \mathrm{E}$, site 3, $2444 \mathrm{~m}: 2{ }^{\curvearrowright}{ }^{\wedge}, 26$ Jan. 2008 (INECN). $-2.87696^{\circ} \mathrm{S}$, $29.49709^{\circ} \mathrm{E}$, site 1, $2650 \mathrm{~m}: 3$ q $q, 25$ Oct. 2008 (INECN).

\section{Other material}

BURUNDI: Parc National de la Kibira, Forêt de Rwegura, pitfalls, B. Nzigidahera coll., 2.90249 ${ }^{\circ}$, $29.51345^{\circ} \mathrm{S}$, forest with Macaranga neomildbreadiana and Xymalos monospora, $2223 \mathrm{~m}, 1$ ○े, 23 Nov. 2003 (INECN).

\section{Description}

Male (holotype, Figs 4-5, 8, 11-13, 26-27, 29)

MEAsurements. Total length 1.70 , carapace 0.70 long, 0.60 wide.

Cephalothorax. Carapace medium brown, with black striae radiating from foveal area. Chelicerae yellow, mottled with grey. Retromargin of chelicerae with 5 teeth; promargin with 4 small teeth. Clypeus 2 times ALE, grey. Sternum dark brown mottled with black, shield-shaped, 0.48 long and 0.44 wide.

EYES. Ocular area black. Posterior eye row strongly procurved, anterior eye row recurved; AME 0.04, ALE 0.08, PME 0.08, PLE 0.08.

LeGs. Pale yellow; tibia I with two spines. Femora without dorsal spines, with ventral spines in two rows. Metatarsi without dorsal spines.

LEG MEASUREMENTS

$\begin{array}{ccccccc}\text { Legs } & \text { Fe } & \text { P } & \text { T } & \text { Mt } & \text { t } & \text { Total } \\ \text { I } & 0.72 & 0.22 & 0.66 & 0.60 & 0.52 & 2.72 \\ \text { II } & 0.70 & 0.22 & 0.62 & 0.54 & 0.50 & 2.58 \\ \text { III } & 0.62 & 0.20 & 0.56 & 0.54 & 0.40 & 2.32 \\ \text { IV } & 0.82 & 0.20 & 0.80 & 0.72 & 0.44 & 2.98\end{array}$



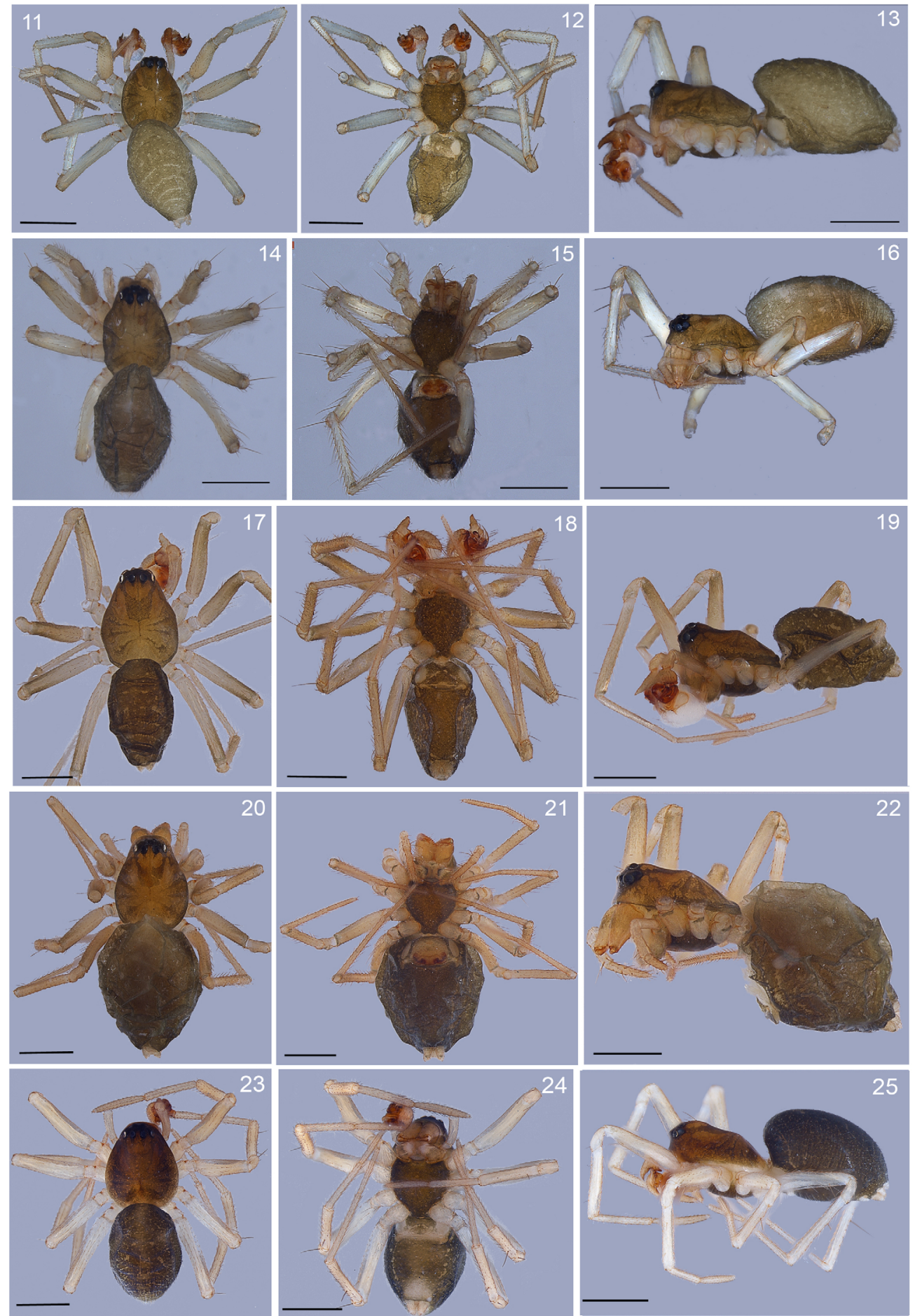

Figs 11-25. - 11-16. Holmelgonia afromontana sp. nov. ( $\circlearrowleft$ holotype, $q$ paratype 227154). 11. Habitus, $\hat{\partial}$, dorsal view. 12. Habitus, $\hat{\partial}$, ventral view. 13. Habitus, $\hat{\partial}$, lateral view. 14. Habitus, $q$, dorsal view. 15. Habitus, $q$, ventral view. 16. Habitus, $q$, lateral view. 17-22. Holmelgonia bosnasutus sp. nov. ( $\delta$ holotype, female paratype 227507). 17. Habitus, $\widehat{\partial}$, dorsal view. 18. Habitus, $\hat{\partial}$, ventral view. 19. Habitus, $\hat{\partial}$, lateral view. 20. Habitus, ${ }^{2}$, dorsal view. 21. Habitus, $q$, ventral view. 22. Habitus, $q$, lateral view. - 23-25. Holmelgonia disconveniens sp. nov. ( $\hat{O}$ holotype). 23. Habitus, dorsal view. 24. Habitus, ventral view. 25. Habitus, lateral view. Scale bars $=0.5 \mathrm{~mm}$. 
LEG CHAETOTAXY

Legs TM

\section{Tibial spines}

\begin{tabular}{lccccc} 
& & \multicolumn{2}{c}{ Proximal } & \multicolumn{2}{c}{ Distal } \\
& & Position & Length/DT & Position & Length/DT \\
I & 0.40 & 0.16 & 1.80 & 0.50 & 1.60 \\
II & 0.20 & 0.14 & 2.20 & 0.42 & 1.40 \\
III & 0.20 & 0.12 & 3.25 & - & - \\
IV & 0.30 & 0.12 & 3.50 & - & -
\end{tabular}

ABDOMEN. Oval, pale grey, dorsally with pale spots and four faint horizontal chevrons becoming smaller towards the rear end. Spinnerets pale yellow, conical.

Palp (Figs 4-5, 8, 26-27, 29). Dorsal tibial apophysis ventrally concave, with tooth-shaped excrescence inside concavity; dorsally with smoothly rounded tip. Paracymbium: proximal arm tapered, with row of short hairs; distal arm with two lobes at extremity, caudal one with some hairs. Prolateral part of subtegulum with oblique grooves. Embolic division (Fig. 29) fairly simple; embolus long, frontal appendage short, truncated, margin with many tiny teeth; dorsal appendage short, rounded.

Female (paratype, MRAC 227154, Figs 14-16, 28, 39)

Habitus similar to male.
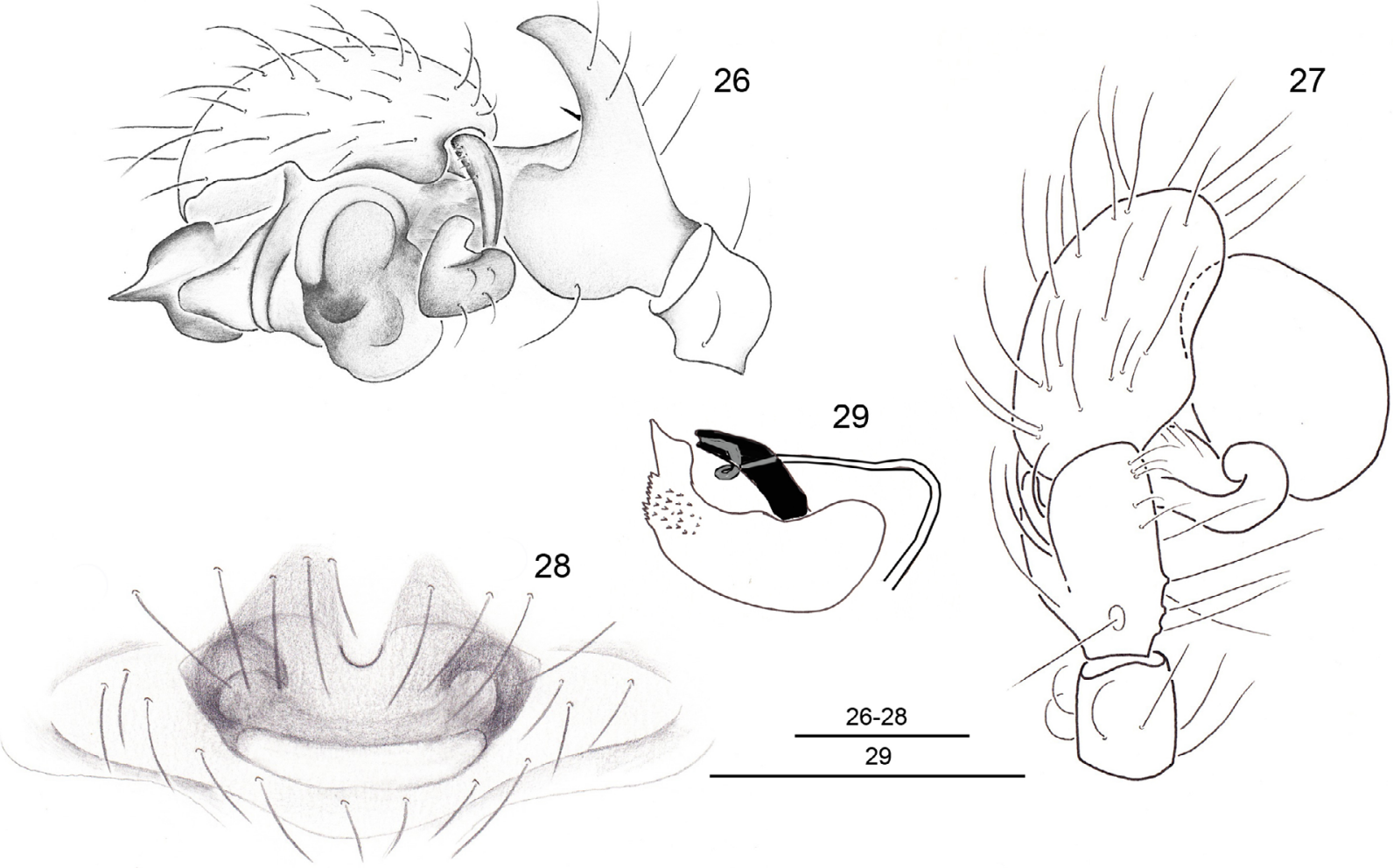

Figs 26-29. Holmelgonia afromontana sp. nov. ( ${ }^{\Uparrow}$ holotype,,+ paratype 227154). 26. Palp, $\widehat{\partial}$, retrolateral view. 27. Palp, $\widehat{\partial}$, dorsal view. 28. Epigyne, ventral view. 29. Embolic division of $\hat{\jmath}$ palp, ventrolateral view. Scale bars $=0.1 \mathrm{~mm}$. 
Measurements. Total length 1.64. Carapace 0.70 long, 0.60 wide.

Cephalothorax. Clypeus 1.66 times ALE. Sternum 0.48 long, 0.46 wide.

EYEs. AME 0.04, ALE 0.08, PME 0.08, PLE 0.08.

$\begin{array}{ccccccc}\text { LEG MEASUREMENTS } & & & & & \\ \text { Legs } & \text { Fe } & \mathbf{P} & \text { T } & \text { Mt } & \mathbf{t} & \text { Total } \\ \text { I } & 0.70 & 0.22 & 0.58 & 0.52 & 0.40 & 2.42 \\ \text { II } & 0.74 & 0.22 & 0.50 & 0.50 & 0.44 & 2.40 \\ \text { III } & 0.64 & 0.20 & 0.44 & 0.44 & 0.36 & 2.08 \\ \text { IV } & 0.80 & 0.20 & 0.66 & 0.66 & 0.46 & 2.78\end{array}$

\section{LEG CHAETOTAXY}

Legs

$\begin{array}{ll} & \\ \text { I } & 0.33 \\ \text { II } & 0.36 \\ \text { III } & 0.40 \\ \text { IV } & 0.54\end{array}$

TM

Proximal

\section{Tibial spines}

\begin{tabular}{cccc}
\multicolumn{2}{c}{ Proximal } & \multicolumn{2}{c}{ Distal } \\
Position & Length/DT & Position & Length/DT \\
0.22 & 2.00 & 0.42 & 1.80 \\
0.10 & 2.00 & 0.38 & 2.00 \\
0.12 & 3.25 & - & - \\
0.16 & 4.00 & - & -
\end{tabular}

EPIGYNe (Fig. 30). Fairly large ventral plate broadly indented in front, posterior margin slightly procurved. Copulatory ducts originate from centre near posterior margin, entering spermathecae near lateral margin; spermathecae large, kidney-shaped, with transverse long axis.

\section{Variation}

Carapace colour may vary from medium brown to yellowish grey.

\section{Distribution}

Known only from the type locality (Fig. 1).

Holmelgonia bosnasutus sp. nov. urn:1sid:zoobank.org:act:098B9E4B-00C0-437E-A36E-EEE46A2D9826

Figs 6, 9, 17-22, 30-34, 40

\section{Diagnosis}

The male is characterized by the sharply pointed dorsal tibial apophysis with undulated margin, each undulation provided with a hair, combined with the broad prolateral apophysis, the broad proximal arm and smoothly rounded tip of the distal arm of the paracymbium. Females are recognized by the shape of the epigyne, provided with two holes clearly visible and opening in the posterior margin.

\section{Etymology}

The specific name 'bosnasutus' refers to the shape of the epigyne which is reminscent of a cow's nose. 


\section{Material examined}

\section{Holotype}

BURUNDI: đ̊, Parc National de la Kibira, Mt. Musumba, Rwegura, 252’ S, $29^{\circ} 30^{\prime}$ E, 28 Dec. 2004, site 2, forest with Hagenia abyssinica, B. Nzigidahera coll. (MRAC 227508).

\section{Paratypes}

BURUNDI: All from Parc National de la Kibira, forêt de Rwegura, pitfalls, B. Nzigidahera coll. $2.89565^{\circ} \mathrm{S}, 29.50345^{\circ} \mathrm{E}$, site 2, forest with Polyscias fulva and Macaranga neomildbraediana, 2120

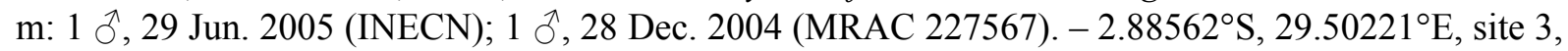
forest with Polyscias fulva and Hagenia abyssinica, $2160 \mathrm{m:} 1$ + , 27 May 2005 (MRAC 227507); 1 ô,

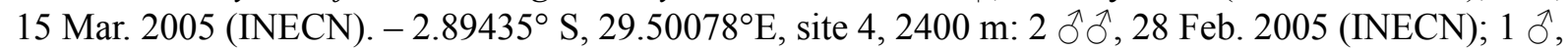
28 Dec. 2004 (INECN).

\section{Other material}

BURUNDI: Parc National de la Kibira, Rwegura, Mt Musumba, B. Nzigidahera coll., 2.86435 ${ }^{\circ}$ S, 29.49540 ${ }^{\circ}$ E, site 7, tea plantation, $2100 \mathrm{~m}: 1$ त, 25 Aug. 2008 (MRAC 227146); 1 §, 10 Jul. 2008

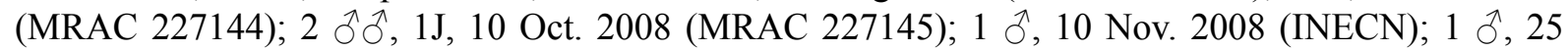
Mar. 2008 (INECN). $-2.86435^{\circ} \mathrm{S}, 29.49546 \mathrm{E}$, site 6, forest with Carapa grandiflora and Polyscias

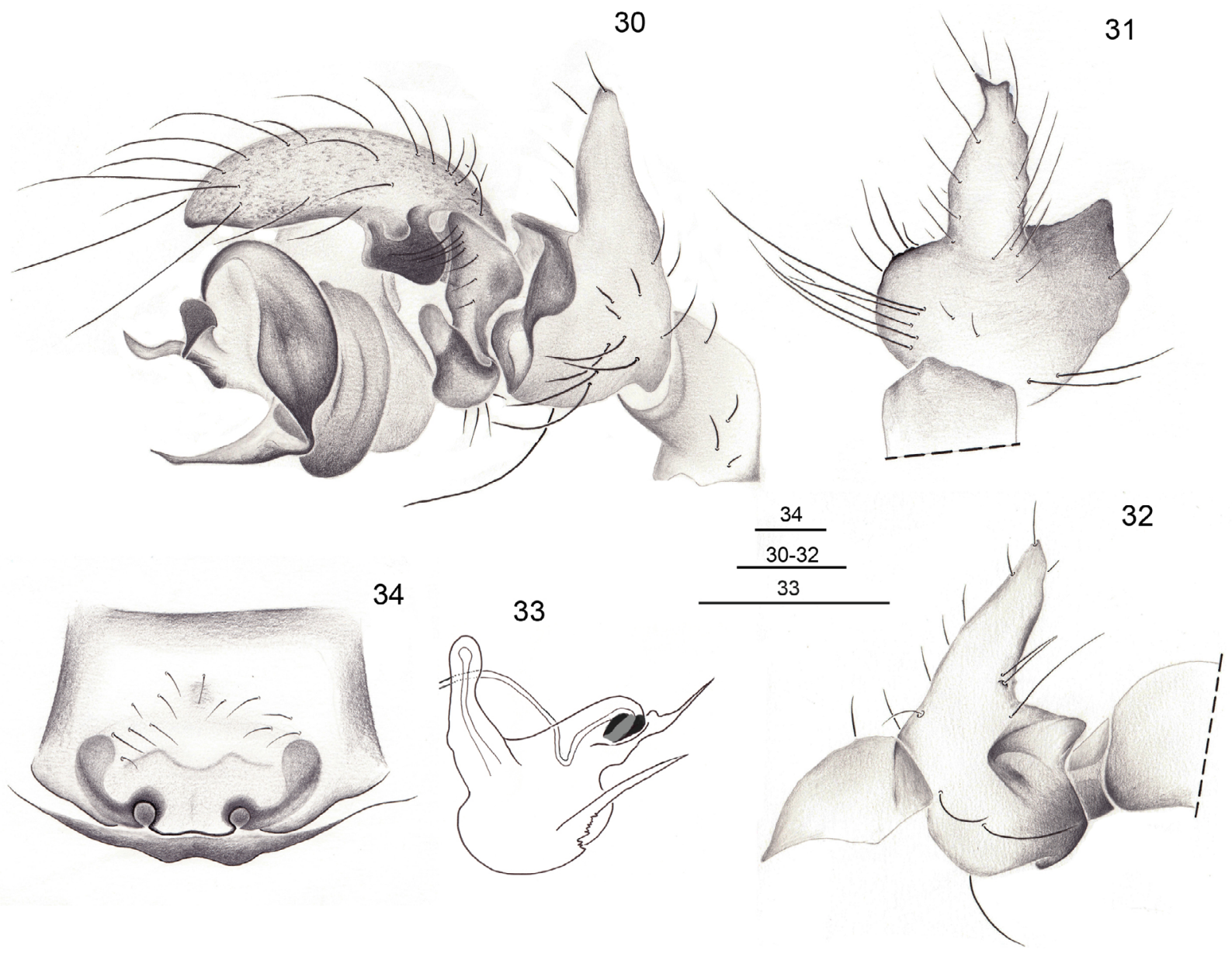

Figs 30-34. Holmelgonia bosnasutus sp. nov. ( $\widehat{\jmath}$ holotype, $q$ paratype 227507). 30. Palp, $\widehat{\partial}$, retrolateral view. 31. Palpal tibia, $\widehat{\partial}$, dorsal view. 32. Palpal tibia, $\hat{\partial}$, prolateral view. 33. Embolic division of $\partial^{\lambda}$ palp, ventrolateral view. 34. Epigyne, ventral view. Scale bars $=0.1 \mathrm{~mm}$. 
fulva, $2150 \mathrm{~m}$ : 1 o, 10 Sep. 2008 (INECN). $-2.86455^{\circ} \mathrm{S}, 29.50427^{\circ} \mathrm{E}$, site 4, forest with Macaranga

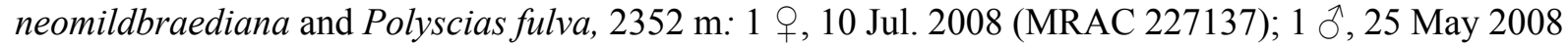

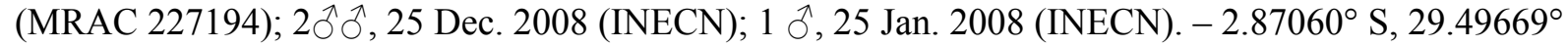
E, site 2, forest with Hagenia abyssinica, $2548 \mathrm{m:} 1$ ○े, 10 Jul. 2008 (MRAC 227195); 1 ○े, 25 Apr. 2008 (MRAC 227196); $1 \overbrace{}^{\lambda}, 25$ Jul. 2008 (INECN); $1 \overbrace{}^{\lambda}, 10$ Sep. 2008 (INECN). - 2.87696º ${ }^{\circ}, 29.49709^{\circ} \mathrm{E}$, site 1, Philippia benguelensis, $2650 \mathrm{~m}: 1$ ภ, 25 Nov. 2008 (INECN).

\section{Description}

Male (holotype, Figs 6, 9, 17-19, 30-33)

MEAsurements. Total length 2.00 , carapace 0.88 long, 0.70 wide.

Cephalothorax. Carapace greyish brown with black striae radiating from fovea; with pale area behind posterior eyes, followed by rectangular spot from which two striae run towards the PLE. Chelicerae coloured as carapace, retromargin with 5 teeth; promargin with 5 small teeth. Clypeus 2.80 times ALE. Sternum 0.54 long, 0.44 wide, dark greyish brown, shield-shaped.

EYES. Ocular area black. Anterior eye row straight, posterior eye row strongly procurved. AME 0.03 , ALE 0.08, PME 0.08, PLE 0.08.

LeGs. Yellow to greyish yellow; femora without dorsal spines, but with ventral spines in two rows. Metatarsi with dorsal spines.

\begin{tabular}{ccccccc}
\multicolumn{2}{c}{ Leg MEASUREMENTS } & & & & & \\
Legs & Fe & $\mathbf{P}$ & T & Mt & $\mathbf{t}$ & Total \\
I & 0.90 & 0.20 & 0.84 & 0.84 & 0.60 & 3.44 \\
II & 0.64 & 0.18 & 0.58 & 0.74 & 0.62 & 2.76 \\
III & 0.60 & 0.16 & 0.60 & 0.64 & 0.54 & 2.54 \\
IV & 0.72 & 0.14 & 0.58 & 0.94 & 0.60 & 2.98
\end{tabular}

\section{LEG CHAETOTAXY}

\section{Legs TM}

\begin{tabular}{lccc} 
& & \multicolumn{2}{c}{ Proximal } \\
& & Position & Length/DT \\
I & 0.38 & 0.18 & 2.75 \\
II & 0.42 & 0.20 & 3.25 \\
III & 0.54 & 0.20 & 3.25 \\
IV & 0.38 & 0.30 & 3.00
\end{tabular}

\section{Tibial spines}

\begin{tabular}{cc}
\multicolumn{2}{c}{ Distal } \\
Position & Length/DT \\
0.75 & 1.75 \\
0.70 & 2.00 \\
- & - \\
- & -
\end{tabular}

Aвdomen. Oval, dark greyish brown, with nine thin, pale transverse lines. Venter dark brown. Spinnerets pale greyish, short, conical.

Palp (Figs 6, 9, 30-33). Retrolateral tibial apophysis with sharp tip and undulated lateral margins, each undulation provided with a hair; laterally with short, broad prolateral apophysis with blunt tip. Paracymbium with broad proximal arm and distal arm with smoothly rounded tip. Proximal part of tegulum with ventrolateral, oblique grooves. Embolic division with short curved embolus, long sharp ventral appendage, sinuous, frontal appendage with sharp tip and long, broad, rounded dorsal appendage. 
Female (paratype, MRAC 227507, Figs 20-22, 34, 40)

Very similar to male.

MeAsurements. Total length 2.13. Carapace 0.90 long, 0.72 wide.

Cephalothorax. Clypeus 3.0 times ALE. Sternum 0.49 long, 0.49 wide.

Eyes. As in male: AME 0.03, ALE 0.08, PME 0.08, PLE 0.08.

LEGS. TM: I: 0.41, II: 0.40, III: 0.50 .

LEG MEASUREMENTS

$\begin{array}{ccccccc}\text { Legs } & \text { Fe } & \text { P } & \text { T } & \text { Mt } & \mathbf{t} & \text { Total } \\ \text { I } & 0.90 & 0.20 & 0.80 & 0.74 & 0.60 & 3.24 \\ \text { II } & 0.66 & 0.12 & 0.54 & 0.68 & 0.60 & 2.60 \\ \text { III } & 0.70 & 0.14 & 0.42 & 0.60 & 0.50 & 2.36 \\ \text { IV } & 0.78 & 0.14 & 0.34 & 0.82 & 0.42 & 2.50\end{array}$

EpIGYNe (Figs 34, 40). With two holes at posterior margin, resembling the shape of a cow's nose. Copulatory ducts short, curved, originating from triangular atrium near posterior margin; entering spermathecae near lateral margin, spermathecae oval, longest axis longitudinal.

\section{Distribution}

Known only from the type locality, Mt Musumba in Kibira National Park (Fig. 1).

Holmelgonia disconveniens sp. nov. urn:1sid:zoobank.org:act:2787FB55-2335-4C64-BFB8-ABC0825A7F56

Figs 2-3, 7, 10, 23-25, 35-38

\section{Diagnosis}

The male is characterized by the triangular dorsal tibial apophysis, with a tooth at the tip and sinuous prolateral margin, by the fairly large, sharp, sclerotized prolateral apophysis, and by the paracymbium without hairs, its distal arm with a well delimited knob.

\section{Etymology}

The specific name 'disconveniens' refers to the contrasting tinge of dark body and pale legs.

\section{Material examined}

\section{Holotype}

BURUNDI: ${ }^{\curvearrowright}$, Parc National de la Kibira, Rwegura, Mt Musumba, $2.86435^{\circ} \mathrm{S}, 29.49540^{\circ} \mathrm{E}$, site 1 , tea plantation, 10 Sep. 2008, 2100 m, pitfalls, B. Nzigidahera coll. (MRAC 229928).

\section{Paratypes}

BURUNDI: 1 đै, 10 Sep. 2008, same data as for holotype (MRAC 229929). $-2.86435^{\circ} \mathrm{S}, 29.49546^{\circ} \mathrm{E}$, site 6, forest with Carapa grandiflora and Polyscias fulva, $2150 \mathrm{~m}: 1 \mathrm{o}^{\top}, 10 \mathrm{Aug} .2008$ (MRAC 229741); $1 \mathrm{O}^{\top}, 10 \mathrm{Sep} .2008$ (MRAC 229740). $-2.86435^{\circ} \mathrm{S}, 29.49540^{\circ} \mathrm{E}$, site 7, tea plantation, $2100 \mathrm{~m}$, site 7: $1 \mathrm{O}^{\top}$,

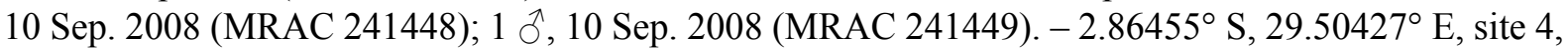




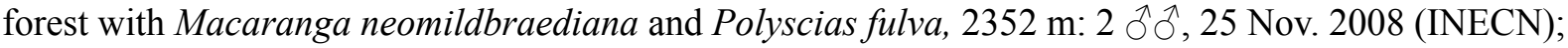

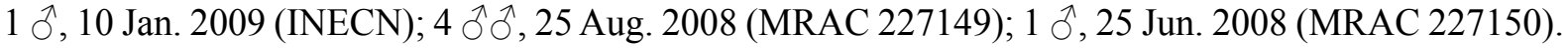

\section{Description}

Male (holotype, Figs 7, 10, 23-25, 35-38)

MeAsurements. Total length 1.8, carapace 0.80 long 0.70 wide.

Cephalothorax. Carapace dark brown, with faint dark striae radiating from fovea; striae larger towards the margin; a faint pale semicircular area behind eyes formed by two brown bands originating behind PLE, connected in front of fovea. Chelicerae yellowish brown. Retromargin with 4 teeth; promargin with 5 small teeth. Clypeus grey, 2.33 times ALE. Sternum 0.56 long, 0.50 wide, dark brown, shieldshaped.

EYES. Ocular area black. Anterior eye row recurved, posterior eye row strongly procurved; AME 0.05 , ALE 0.08, PME 0.07, PLE 0.07.

Legs. Pale yellow, tarsi and metatarsi pale grey, femora without dorsal spines, with ventral spines in two rows. Metatarsi without dorsal spines.
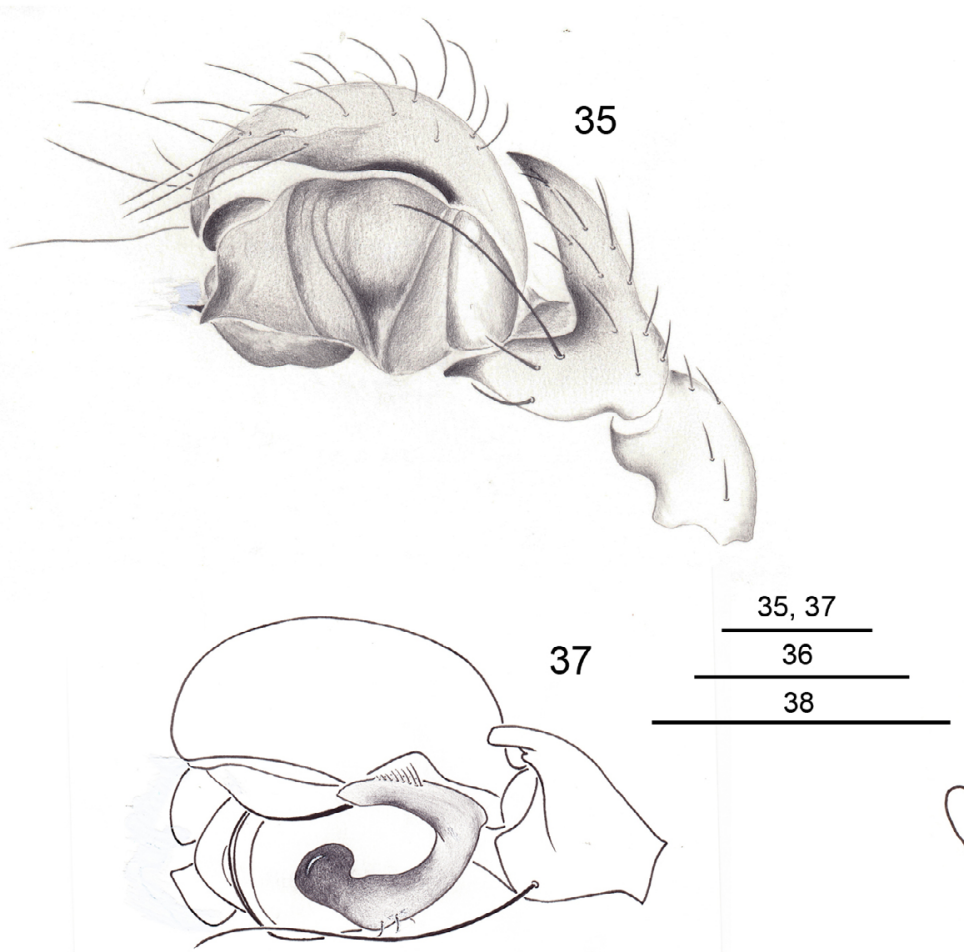

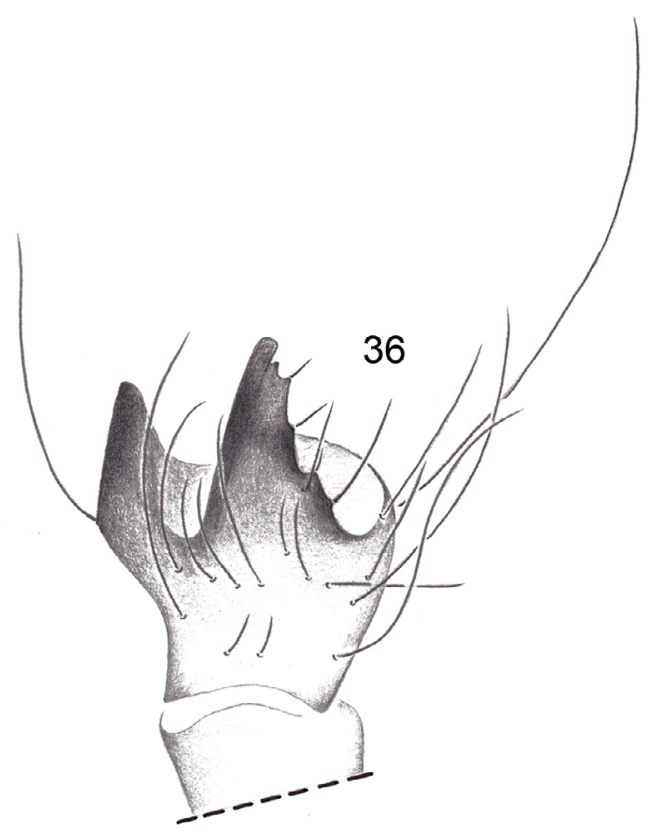

38

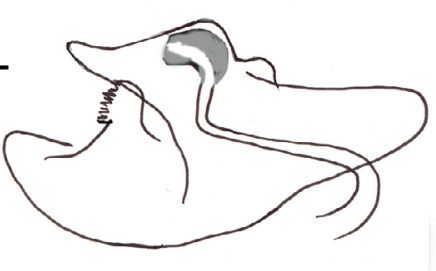

Figs 35-38. Holmelgonia disconveniens sp. nov. ( $\sigma^{\Uparrow}$ holotype). 35. Palp, retrolateral view. 36. Palpal tibia, dorsal view. 37. Palpal tibia, dorsolateral view. 38. Embolic division of palp, ventrolateral view. Scale bars $=0.1 \mathrm{~mm}$. 


$\begin{array}{ccccccc}\text { LEG MEASUREMENTS } & & & & & \\ \text { Legs } & \text { Fe } & \mathbf{P} & \text { T } & \text { Mt } & \text { t } & \text { Total } \\ \text { I } & 0.70 & 0.22 & 0.58 & 0.56 & 0.48 & 2.54 \\ \text { II } & 0.66 & 0.22 & 0.56 & 0.56 & 0.42 & 2.42 \\ \text { III } & 0.60 & 0.20 & 0.50 & 0.50 & 0.52 & 2.32 \\ \text { IV } & 0.76 & 0.22 & 0.64 & 0.70 & 0.56 & 2.88\end{array}$

LEG CHAETOTAXY

Legs TM

Tibial spines

\begin{tabular}{lccccc} 
& & \multicolumn{2}{c}{ Proximal } & \multicolumn{2}{c}{ Distal } \\
& & Position & Length/DT & Position & Length/DT \\
I & 0.55 & 0.12 & 0.60 & 0.40 & 1.00 \\
II & 0.42 & 0.18 & 0.60 & 0.44 & 1.00 \\
III & 0.40 & 0.12 & 3.00 & - & - \\
IV & 0.45 & 0.12 & 3.25 & - & -
\end{tabular}

AвDomen. Oval, greyish black, with faint central dark brown band and small pale points, with six transverse pale chevrons in posterior half. Spinnerets yellowish grey, conical.

PaLP (Figs 7, 10, 35-38). Dorsal tibial apophysis triangular, with two teeth near tip; retrolateral tibial apophysis almost as long as dorsal one, ending in sharp, slightly curved tip. Paracymbium with proximal arm without hairs; distal arm ending in well delimited, rounded knob. Subtegulum with transverse ventral grooves. Embolic division with short, curved embolus, posterior appendage very short and smoothly rounded; dorsal appendage triangular; frontal appendage short, club-shaped, with frontal margin with many small teeth.

\section{Female}

Unknown.

\section{Distribution}

Known only from the type locality (Fig. 1).
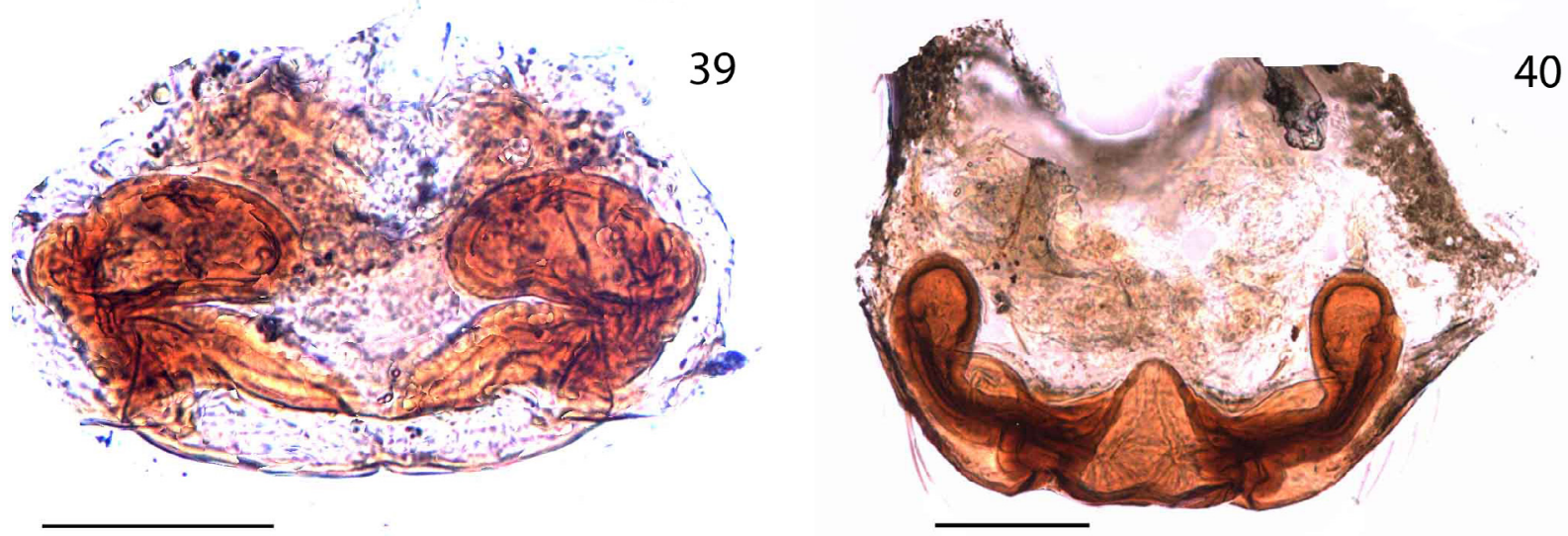

Figs 39-40. - 39. Holmelgonia afromontana sp. nov. ( + , 227154). Epigyne, cleared, dorsal view. 40. Holmelgonia bosnasutus sp. nov. (q, 227507). Epigyne, cleared, dorsal view. Scale bars $=0.1 \mathrm{~mm}$. 


\section{Discussion}

Linyphiidae are a striking example of a family in which genera have been erected based on secondary sexual characters that appear to be species specific. This phenomenon was discussed in Jocqué et al. (2013), who argued that genera in some families are particularly difficult to define when they contain so-called 'templates' (as defined by Jocqué \& Bosselaers 2011), lacking clear somatic autapomorphies. The definition of Holmelgonia is no exception to this: apart from the absence of stridulating files on the chelicerae, no somatic characters could be found to define it. Even the chaetotaxy, and more specifically the position of the metatarsal trichobothria, varies to an extent that is unusual in erigonine genera. The genus has therefore to be defined on the base of features of the secondary genitalia. The palp has a tibia with a dorsal process and the ED is rather complex, with several protuberances. Yet, in two of the species described here the ED is fairly simple. The latter feature, however, is not restricted to Holmelgonia and can be found in many genera in the Erigoninae. It thus remains questionable whether the species attributed to Holmelgonia really do constitute a natural group.

This study is a result of the intense sampling of ground spiders carried out in the forest ecosystems of western Burundi during several years (2003, 2005 and 2008). The three species described here were only found in the mountain forest of Kibira National Park. Holmelgonia bosnasutus sp. nov. occupies a wide altitudinal gradient, ranging from 2100 to $2650 \mathrm{~m}$. The species $H$. afromontana sp. nov. and $H$. disconveniens sp. nov. are confined to a narrower altitudinal zone between 2100 and $2352 \mathrm{~m}$.

This study shows again that the Albertine Rift in Central Africa has only been superficially explored with regard to its spider fauna. This was already clear with the discovery of species swarms in the Gnaphosidae, in which the genera Zelotibia Russell-Smith \& Murphy, 2005 (20 species) and Zelowan Murphy \& Russell-Smith, 2010 (18 species) were found to have radiated in that mountain range (RussellSmith \& Murphy 2005; Murphy \& Russell-Smith 2010; Nzigidahera \& Jocqué 2009). Further studies in preparation show that similar radiations can be expected in other genera of the Linyphiidae and in other families such as the Tetragnathidae, particularly in ground-living genera that have lost the web-building habit. There is a considerable urgency to describe the fauna in this region, where human populations are expanding rapidly and deforestation is consequently very intense.

\section{Acknowledgements}

ABIC (African Biodiversity Information Centre) of the Royal Museum for Central Africa and GBIF are thanked for several travel grants to BN. We are indebted to the Royal Museum for Central Africa for accommodation for BN during his studies in Belgium. We thank Alain Reygel, who made the drawings with his usual skill. We thank two anonymous reviewers for improvements to the first version.

\section{References}

Frick H. \& Scharff N. 2013. Phantoms of Gondwana? - phylogeny of the spider subfamily Mynogleninae (Araneae: Linyphiidae). Cladistics 29: 1-40. http://dx.doi.org/10.1111/cla.12025

Holm $\AA$. 1962. The spider fauna of the East African mountains. Part I: Fam. Erigonidae. Zoologiska Bidrag från Uppsala 35: 19-204.

Jocqué R., Alderweireldt M. \& Dippenaar-Schoeman A. 2013. Biodiversity, an African perspective. In: Penney D. (ed.) Spider Research in the 21st Century: 18-57. Siri Scientific Press. Rochdale, U.K.

Jocqué R. \& Bosselaers J. 2011. Revision of Pseudocorinna Simon and a new related genus (Araneae: Corinnidae): two more examples of spider templates with a large range of complexity in the genitalia. Zoological Journal of the Linnean Society 162: 271-350. http://dx.doi.org/10.1111/j.1096$\underline{3642.2010 .00679 . x}$ 
Jocqué R. \& Scharff N. 1986. Spiders of the family Linyphiidae (Araneae) from the Tanzanian mountain areas Usambara, Uluguru and Rungwe. Annales du Musée royal de l'Afrique Centrale, Sciences Zoologiques 248: 1-61.

Jocqué R. \& Scharff N. 2007. Holmelgonia, a new name for the genus Elgonia Holm, 1989 (Araneae, Linyphiidae). Journal of Afrotropical Zoology 3: 161.

Murphy J.A. \& Russell-Smith A. 2010. Zelowan, a new genus of African zelotine ground spiders (Araneae: Gnaphosidae). Journal of Afrotropical Zoology 6: 59-82.

Nzigidahera B. \& Jocqué R. 2009. An update of Zelotibia (Araneae, Gnaphosidae), a spider genus with a species swarm in the Albertine Rift. ZooKeys 13: 1-28. http://dx.doi.org/10.3897/zookeys.13.145

Platnick N.I. 2013. The World Spider Catalog. Version 13.5. American Museum of Natural History. Available from http://research.amnh.org/iz/spiders/catalog/ (accessed 20 January 2013).

Russell-Smith A. \& Murphy J.A. 2005. Zelotibia, a new zelotine spider genus from central Africa (Araneae, Gnaphosidae). Journal of Afrotropical Zoology 2: 103-122.

Scharff N. 1990. Spiders of the family Linyphiidae from the Uzungwa Mountains, Tanzania (Araneae). Entomologica Scandinavica Supplements 36: 1-95.

Scharff N. 1992. The linyphiid spider fauna of eastern Africa (Araneae: Linyphiidae)-distribution patterns, diversity and endemism. Biological Journal of the Linnean Society 45: 117-54. http://dx.doi. org/10.1111/j.1095-8312.1992.tb00635.x

Scharff N. 1993. The Linyphiid spider fauna (Araneae: Linyphiidae) of mountain forests in the Eastern Arc mountains. In: Lovett J.C. \& Wasser S.K. (eds) Biogeography and Ecology of the Rain Forests of Eastern Africa: 115-132. Cambridge University Press.

Seyfulina R.R. \& Jocqué R. 2007. An interactive key to the Afrotropical genera of Linyphiidae. http:// www.africamuseum.be/research/natural-sciences/biology/invertebrates-ni/collections/intkey

Seyfulina R.R. \& Jocqué R. 2009. Venia kakamega gen. n., sp. n., a new, canopy-dwelling Afrotropical erigonine spider (Araneae, Linyphiidae). Journal of Afrotropical Zoology 5: 3-13.

Manuscript received: 5 September 2013

Manuscript accepted: 8 November 2013

Published on: 17 March 2014

Topic editor: Koen Martens

Desk editor: Danny Eibye-Jacobsen

Printed versions of all papers are also deposited in the libraries of the institutes that are members of the EJT consortium: Muséum National d'Histoire Naturelle, Paris, France; National Botanic Garden of Belgium, Meise, Belgium; Royal Museum for Central Africa, Tervuren, Belgium; National History Museum, London, United Kingdom; Royal Belgian Institute of Natural Sciences, Brussels, Belgium; Natural History Museum of Denmark, Copenhagen, Denmark. 